\title{
Concordancia entre histología pre, intra y postoperatoria en cáncer de endometrio
}

\author{
Felipe Serman V. ${ }^{1}$, María Elena Sánchez $A .{ }^{1}$, Bárbara Barrientos $F^{2}{ }^{2}$, Bárbara Saldaña \\ G. ${ }^{3}$, Javier Trullen S. ${ }^{a}$, Daniela Burky A. a, Paula Calvo S.a, Maritza Matus I.b, Roderick \\ Walton L. 4 \\ ${ }^{1}$ Unidad de Ginecología Oncológica, ${ }^{2}$ Servicio de Anatomía Patológica, ${ }^{3}$ Servicio de Radioterapia, Hospital de Carabi- \\ neros de Chile. ${ }^{4}$ Facultad de Ciencias Médicas, Universidad de Santiago.
}

a Alumnos, Escuela de Medicina, Universidad Mayor. ${ }^{b}$ Matrona.

\section{RESUMEN}

Antecedentes: En pacientes con cáncer de endometrio se discute la concordancia entre biopsia pre e intraoperatoria versus la definitiva, en grado de diferenciación, tipo histológico y profundidad de mioinvasión. Método: Se determinó sensibilidad (S), especificidad (E), valor predictivo positivo (VPP) y valor predictivo negativo (VPN) de: grado de diferenciación bien diferenciado y tipo histológico sólo endometrioide en biopsia preoperatoria; y grado de diferenciación bien diferenciado, tipo histológico sólo endometrioide y profundidad de mioinvasión menor o igual a la mitad interna del miometrio, en biopsia intraoperatoria, para resultados similares en biopsia definitiva en serie clínica de pacientes con cáncer de endometrio clínicamente limitado al útero sometidas a tratamiento quirúrgico primario. Resultados: Biopsia preoperatoria: grado de diferenciación bien diferenciado S: 100\%, E: 77,8\%, VPP: 78,9\% y VPN: 100\%. Tipo histológico sólo endometrioide S: $86,4 \%$, E: $33,3 \%$, VPP: $86,4 \%$ y VPN: $33,3 \%$, para resultado similar en biopsia definitiva. Biopsia intraoperatoria: grado de diferenciación bien diferenciado S: $91,7 \%$, E: $94,4 \%$, VPP: $91,7 \%$ y VPN: $100 \%$. Tipo histológico sólo endometrioide S: 95,7\%, E: 77,8\%, VPP: 95,7\% y VPN: 70\%. Profundidad de mioinvasión menor o igual a mitad interna del miometrio S: $95,2 \%$, E: $30 \%$, VPP: $74,1 \%$ y VPN: $75 \%$, para resultado similar en la biopsia definitiva. Conclusión: No hubo concordancia absoluta de grado de diferenciación bien diferenciado entre biopsia preoperatoria y biopsia definitiva, ni de grado de diferenciación bien diferenciado ni tipo histológico sólo endometrioide entre biopsia intraoperatoria y biopsia definitiva, por lo que se recomienda etapificación quirúgica independiente de los resultados de las biopsias pre e intra operatoria.

\section{PALABRAS CLAVE: Cáncer de endometrio, precisión de la biopsia, etapificación quirúrgica}

\section{SUMMARY}

Background: Among patients with endometrial cancer there is discussion about concordance between pre and intraoperatory biopsy and the definitive one, for diferentiation grade, histologyc type and depth of myoinvasion. Method: To determinate sensibility (S), specificity (Sp), positive predictive value (PPV) and negative predictive value (NPV) of: good diferenciated diferentiation grade and only endometrioid histologyc type on preoperatory biopsy, and good diferenciated diferentiation grade, only endometrioid histologyc type and inner half of the miometryum or less myoinvasion depth on intraoperatory biopsy, for similar result on definitive biopsy in a clinical cohort of patients with endometrial cancer clinically limited to uterus treated with primary surgery. Results: Preoperatory biopsy: good diferentiated diferentiation grade S: $100 \%$, Sp: $77.8 \%$, 
PPV: $78.9 \%$ and NPV: $100 \%$. Only endometrioid histologyc type S: $86.4 \%$, Sp: $33.3 \%$, PPV $86.4 \%$ and NPV $33.3 \%$, for similar result on definitive biopsy. Intraoperatory biopsy: Good diferentiated diferentiation grade S: $91.7 \%$, Sp: $94.4 \%$, PPV: $91.7 \%$ and NPV 100\%. Only endometrioid histologyc type S: $95.7 \%$, Sp $77.8 \%$, PPV $95.7 \%$ and NPV 70\%. Inner half of the myometrium or less myoinvasion depth S: $95.2 \%$, Sp 30\%, PPV $74.1 \%$ and NPV $75 \%$, for similar result on definitive biopsy. Conclusion: There wasn't absolute concordance of good diferentiated diferentiation grade between preoperatory and definitive biopsy. Also there weren't absolute concordance of neither good diferentiated diferentiation grade, nor only endometrioid histologyc type, between intraoperatory and definitive biopsy, for that it is recommended surgical staging, independent of results of pre and intraoperatory biopsies.

\section{KEY WORDS: Endometrial cancer, biopsy accuracy, surgical staging}

\section{INTRODUCCIÓN}

Existe evidencia que revela que en pacientes con cáncer de endometrio clínicamente limitado al útero, y sometidas a tratamiento quirúrgico primario, no existe concordancia absoluta entre el grado de diferenciación $(G)$ del tejido tumoral obtenido preoperatoriamente por legrado uterino o por biopsia endometrial ambulatoria, y el $\mathrm{G}$ del tumor uterino determinado en la biopsia definitiva, ni entre el tipo histológico $(\mathrm{TH})$ del tejido tumoral obtenido preoperatoriamente por legrado uterino o por biopsia endometrial ambulatoria, y el TH del tumor presente en el útero en la biopsia definitiva. Además, existe evidencia de que tampoco hay concordancia absoluta entre el $\mathrm{G}$ del tumor determinado en la biopsia intraoperatoria y el $\mathrm{G}$ del tumor determinado en la biopsia definitiva, ni entre el TH del tumor determinado en la biopsia intraoperatoria y el TH del tumor determinado en la biopsia definitiva. Tampoco entre la profundidad de mioinvasión (PMI) del tumor determinada en la biopsia intraoperatoria y la PMI del tumor determinada en la biopsia definitiva (1-5). Se ha considerado que en pacientes con cáncer de endometrio clínicamente limitado al útero, bien diferenciado (G1), de tipo histológico endometrioide, y con profundidad de mioinvasión de hasta la mitad interna del miometrio, existiría baja incidencia de diseminación linfonodal, por lo que bastaría con la constatación de estos parámetros en la biopsia intraoperatoria según algunos autores $(6,7)$, para abstenerse de realizar la etapificación quirúrgica completa, cuyo componente esencial es la linfadenectomía pélvica y aórtica.

Desde la aparición en nuestro medio de la recomendación de usar la biopsia por congelación para decidir si realizar o no etapificación quirúrgica completa en pacientes con tratamiento quirúrgico primario por cáncer de endometrio (6), se inició este abordaje en nuestro Servicio, cuya evaluación es motivo del presente trabajo.

El objetivo de este estudio es determinar en pacientes con diagnóstico de cáncer de endometrio clínicamente limitado al útero:

a) la concordancia del grado de diferenciación y tipo histológico tumoral entre la histopatología preoperatoria y el estudio histopatológico definitivo del útero.

b) la concordancia del grado de diferenciación, tipo histológico y profundidad de mioinvasión tumoral entre la biopsia intraoperatoria y el estudio histopatológico definitivo del útero.

\section{MATERIAL Y MÉTODO}

Construcción de base de datos de 92 pacientes en quienes se realizó tratamiento quirúrgico primario por cáncer de endometrio clínicamente limitado al útero, en la Unidad de Ginecología Oncológica del Hospital de Carabineros de Chile, entre 1991 y 2010. Se consignaron las siguientes variables:

1. En la biopsia preoperatoria: a) tipo histológico $(T H)$, b) grado de diferenciación $(G)$ en las pacientes con tipo histológico endometrioide.

2. En la biopsia intraoperatoria (cuando ésta fue realizada): a) $\mathrm{TH}$, b) $\mathrm{G}$ en las pacientes con $\mathrm{TH}$ endometrioide. c) Profundidad de la mioinvasión (PMI) en las pacientes con TH endometrioide.

3. En la biopsia definitiva: idem a, b y c.

Se define como biopsia definitiva la evaluación anátomo-patológica final en el espécimen de la histerectomía (estudio histopatológico definitivo del útero).

Determinación de la concordancia entre los siguientes parámetros:

a) G pre y postoperatorio e intra y postoperatorio en pacientes con $\mathrm{TH}$ endometrioide.

b) TH pre y postoperatorio e intra y postoperatorio.

c) Profundidad de mioinvasión (PMI) en la biopsia intraoperatoria y en la biopsia definitiva en las pa- 
cientes con TH endometrioide mediante: cálculo de sensibilidad (S), especificidad (E), valor predictivo positivo (VPP) y valor predictivo negativo (VPN), según fórmulas de Altman $(8,9)$ de:

a) G1 y TH sólo endometrioide preoperatorios (PO) para resultado similar en biopsia definitiva (BD).

b) G1, TH sólo endometrioide y $\mathrm{PMI}$ menor o igual a la mitad interna del miometrio y de biopsia intraoperatoria $(\mathrm{BIO})$ para resultado similar en biopsia definitiva (BD) (en pacientes en quienes se realizó biopsia intraoperatoria).

Para a) y b) se definió verdaderos positivos y verdaderos negativos sólo cuando el $\mathrm{G}$ y $\mathrm{TH}$ de la biopsia preoperatoria (a) coincidían con los de la biopsia definitiva, y sólo cuando G, TH y PMI de la biopsia intraoperatoria coincidían con los de la biopsia definitiva (b). De otra manera, los casos fueron definidos como falsos positivos y falsos negativos de $\mathrm{G}$ preoperatorio, TH preoperatorio, G de biopsia intraoperatoria, TH de biopsia intraoperatoria, o PMI de biopsia intraoperatoria.

Se analizó la significancia estadística de las diferencias entre los valores de los parámetros referidos entre biopsia pre e intraoperatoria versus definitiva mediante test de ji cuadrado $\left(x^{2}\right)$ con corrección de Yates.

\section{RESULTADOS}

De las 92 pacientes con diagnóstico de cáncer de endometrio que recibieron tratamiento quirúrgico primario, en 48 el tipo histológico en la biopsia definitiva fue sólo endometrioide. En 33 de ellas se tuvo información del G preoperatorio. En 19 de ellas el G de la biopsia preoperatoria fue $\mathrm{G} 1$, mientras que en la biopsia definitiva, sólo en 15 de éstas el G fue G1 (Tabla I). El grado de diferenciación G1 en biopsia preoperatoria tuvo una sensibilidad de $100 \%$, especificidad de $77,8 \%$, VPP de $78,9 \%$ y VPN de $100 \%$ para G1 en biopsia definitiva (Tabla II).

\section{Tabla I}

\section{RESULTADOS DE GRADO TUMORAL POR BIOPSIA PREOPERATORIA Y BIOPSIA DEFINITIVA}

\begin{tabular}{lcc}
\hline & G1 en BD & $G>1$ en $B D$ \\
\hline G1PO & 15 & 4 \\
$G>1$ PO & 0 & 14
\end{tabular}

G: grado diferenciación. PO: biopsia preoperatoria. BD: biopsia definitiva. $x^{2}=16,33$; $p<0,0001$
Tabla II

S, E, VPP Y VPN DE DISTINTOS PARÁMETROS HISTOLÓGICOS PRE E INTRAOPERATORIOS PARA RESULTADO SIMILAR EN HISTOLOGÍA UTERINA DEFINITIVA EN CÁNCER DE ENDOMETRIO

\begin{tabular}{lcccc}
\hline & S\% & E\% & VPP\% & VPN\% \\
\hline G1PO & 100 & 77,8 & 78,9 & 100 \\
THSEPO & 86,4 & 33,3 & 86,4 & 33,3 \\
G1IO & 91,7 & 94,4 & 91,7 & 100 \\
THSEIO & 93,8 & 77,8 & 95,7 & 70,0 \\
PMI $\leq$ MIIO & 95,2 & 30,0 & 74,1 & 75,0 \\
\hline
\end{tabular}

G1PO: G1 en biopsia preoperatoria. THSEPO: tipo histológico sólo endometrioide en biopsia preoperatoria. G1IO: G1 en biopsia intraoperatoria. THSEIO: tipo histológico sólo endometrioide en biopsia intraoperatoria. PMI $\leq$ MIIO: profundidad de mioinvasión $\leq$ a la mitad interna del miometrio en biopsia intraoperatoria. S: sensibilidad. E: especificidad. VPP: valor predictivo positivo. VPN: valor predictivo negativo.

De las 92 pacientes, en 53 hubo información del tipo histológico preoperatorio. De las 44 pacientes en que el tipo histológico fue sólo endometrioide en la biopsia preoperatorio, sólo en 38 de ellas se mantuvo este tipo histológico exclusivo en la biopsia definitiva (Tabla III). El tipo histológico sólo endometrioide, en biopsia preoperatoria tuvo sensibilidad de $86,4 \%$, especificidad de $33,3 \%$, VPP de $86,4 \%$ y VPN de $33,3 \%$ para tipo histológico similar en biopsia definitiva (Tabla II).

\section{Tabla III \\ RESULTADOS DE TIPO HISTOLÓGICO POR BIOPSIA PREOPERATORIO Y BIOPSIA DEFINITIVA}

\begin{tabular}{|c|c|c|}
\hline & $\begin{array}{l}\text { TH sólo } \\
\text { endometrioide } \\
\text { en } \mathrm{BD}\end{array}$ & $\begin{array}{l}\text { TH no } \\
\text { endometrioide } \\
\text { en BD }\end{array}$ \\
\hline $\begin{array}{l}\text { TH sólo } \\
\text { endometrioide } \\
\text { en PO }\end{array}$ & 38 & 6 \\
\hline $\begin{array}{l}\text { TH no } \\
\text { endometrioide } \\
\text { en PO }\end{array}$ & $\begin{array}{l}6 \\
1 \text { mucinoso } \\
1 \text { células claras } \\
2 \text { mixtos } \\
2 \text { indiferenciados }\end{array}$ & $\begin{array}{l}3 \\
1 \text { seroso papilar } \\
1 \text { células claras } \\
1 \text { mixto }\end{array}$ \\
\hline
\end{tabular}

TH: tipo histológico. PO: biopsia preoperatoria. BD: biopsia definitiva. $x^{2}=0,90 ; p=0,3437$ 
En 57 pacientes hubo biopsia uterina intraoperatoria. Se consignó el $\mathrm{G}$ en 29 de ellas con tipo histológico endometrioide. De éstas, en 12 el $\mathrm{G}$ fue $\mathrm{G} 1$, y en 11 el $\mathrm{G}$ fue $>1$. En la biopsia definitiva, de las $\mathrm{G} 1$ en la biopsia intraoperatoria, en 11 se mantuvo este grado de diferenciación (Tabla IV). Las pacientes G1 en biopsia intraoperatoria tuvieron una sensibilidad de $91,7 \%$, especificidad de $94,4 \%$, VPP de $91,7 \%$ y VPN de $100 \%$ para G1 en biopsia definitiva (Tabla II).

Tabla IV

\section{RESULTADOS DE GRADO TUMORAL POR BIOPSIA INTRAOPERATORIA Y BIOPSIA DEFINITIVA}

\begin{tabular}{lcc}
\hline & G1 en BD & $G>1$ en BD \\
\hline$G 1$ en BIO & 11 & 1 \\
$G>1$ en BIO & 0 & 17
\end{tabular}

G: grado diferenciación. BIO: biopsia intraoperatoria. BD: biopsia definitiva. $x^{2}=21,36 ; p<0,00001$

En 57 pacientes se consignó el tipo histológico en la biopsia intraoperatoria. En 47 de ellas, éste fue sólo endometrioide. De éstas, en 45 se mantuvo el tipo histológico en la biopsia definitiva (Tabla V). El tipo histológico sólo endometrioide en biopsia intraoperatoria tuvo sensibilidad de $93,8 \%$, especificidad de $77,8 \%$, VPP de $95,7 \%$ y VPN de $70 \%$ para tipo histológico sólo endometroide en biopsia definitiva (Tabla II).

En 31 pacientes con tipo histológico endometrioide se consignó la PMI en la biopsia intraoperatoria. En 27 de ellas, ésta fue menor o igual a

Tabla V

TIPO HISTOLÓGICO SEGÚN BIOPSIA INTRAOPERATORIA Y BIOPSIA DEFINITIVA

\begin{tabular}{lcc}
\hline & $\begin{array}{c}\text { TH sólo } \\
\text { endometrioide } \\
\text { en BD }\end{array}$ & $\begin{array}{c}\text { TH no } \\
\text { endometrioide } \\
\text { en BD }\end{array}$ \\
\hline $\begin{array}{l}\text { TH sólo } \\
\text { endometrioide } \\
\text { en BIO }\end{array}$ & 45 & 2 \\
$\begin{array}{l}\text { TH no } \\
\text { endometrioide } \\
\text { en BIO }\end{array}$ & 3 & 7 \\
\hline
\end{tabular}

TH: tipo histológico. BIO: biopsia intraoperatoria. BD: biopsia definitiva. $x^{2}=22,09 ; p<0,00001$. la mitad interna del miometrio. Sin embargo, en la biopsia definitiva, sólo en 20 de ellas la profundidad de la mioinvasión fue menor o igual a la mitad interna del miometrio, invadiendo en las restantes 7 la mitad externa (Tabla VI). PMI menor o igual a la mitad interna del miometrio en la biopsia intraoperatoria tuvo sensibilidad de $95,2 \%$, especificidad de $30 \%$, VPP de $74,1 \%$ y VPN de $75 \%$ para profundidad de invasión menor o igual a la mitad interna del miometrio en la biopsia definitiva, en las pacientes con tipo histológico endometrioide (Tabla II).

\section{Tabla VI \\ PROFUNDIDAD DE MIOINVASIÓN SEGÚN BIOPSIA INTRAOPERATORIA Y BIOPSIA DEFINITIVA}

\begin{tabular}{lcc}
\hline & $\begin{array}{c}\text { PMI } \leq \text { a mitad } \\
\text { interna del } \\
\text { miometrio en } \\
\text { BD }\end{array}$ & $\begin{array}{c}\text { PMI > mitad } \\
\text { interna del } \\
\text { miometrio en } \\
\text { BD }\end{array}$ \\
\hline $\begin{array}{l}\mathrm{PMI} \leq \text { a mitad } \\
\text { interna } \\
\text { del miometrio en }\end{array}$ & 20 & 7 \\
BIO & & \\
$\begin{array}{l}\text { PMI > mitad } \\
\text { interna } \\
\text { del miometrio en }\end{array}$ & 1 & 3 \\
BlO & & \\
\hline
\end{tabular}

PMI: profundidad de mioinvasión. BIO: biopsia intraoperatoria. $\mathrm{BD}$ : biopsia definitiva. $x^{2}=1,92 ; \mathrm{p}<0,1656$.

\section{DISCUSIÓN}

En esta serie de pacientes con cáncer de endometrio clínicamente limitado al útero sometidas a tratamiento quirúrgico primario, se logró determinar sensibilidad, especificidad, valor predictivo positivo y valor predictivo negativo de los siguientes parámetros para resultado similar en el espécimen histopatológico definitivo: grado de diferenciación G1 en biopsia preoperatoria, tipo histológico sólo endometrioide en biopsia preoperatoria, grado de diferenciación G1 en biopsia intraoperatoria, tipo histológico sólo endometrioide en biopsia intraoperatoria, profundidad de mioinvasión menor o igual a la mitad interna del miometrio en biopsia intraoperatoria.

En nuestra casuística se observa que no hay concordancia absoluta entre grado de diferenciación bien diferenciado (G1) en la biopsia preoperatoria y $G$ similar en la biopsia definitiva (S: $100 \%$, 
E: 77,8\%, VPP: 78,9\%, VPN: $100 \%)(p<0,0001) \mathrm{ni}$ entre $\mathrm{G} 1$ en la biopsia intraoperatoria y $\mathrm{G} 1$ en la biopsia definitiva (S: 91,7\%, E: 94,4\%, VPP: 91,7\%, VPN: $100 \%)(p<0,00001)$. Más importante aún, no existe concordancia absoluta en tipo histológico sólo endometrioide entre la biopsia intraoperatoria y la biopsia definitiva (S: 93,8\%, E: 77,8\%, VPP: 95,7\%, VPN: $70 \%)(p<0,00001)$.

Estas diferencias alcanzaron significancia estadística en los siguientes parámetros: $\mathrm{G} 1$ en biopsia preoperatoria vs G similar en biopsia definitiva, G1 en biopsia intraoperatoria vs G1 en biopsia definitiva, y tipo histológico sólo endometrioide en biopsia intraoperatoria vs tipo histológico sólo endometrioide en biopsia definitiva.

En pacientes con cáncer de endometrio, la presencia de tipo histológico endometrioide con grado de diferenciación mayor que 1 , tipo histológico no endometrioide, o profundidad de invasión mayor que la mitad interna del miometrio han demostrado en distintas series $(4,10,11,12,13)$ incidencia significativa de enfermedad extrauterina microscópica, esencialmente a nivel linfonodal pélvico y lumboaórtico.

Es necesario considerar que los factores históricos de riesgo de compromiso linfonodal en cáncer de endometrio, de acuerdo a un trabajo fundamental del GOG (10), están estudiados en biopsia definitiva, no en biopsia por congelación. Además, un factor relevante para el riesgo de diseminación linfonodal, y para sobrevida, como lo es el compromiso microscópico de espacios linfovasculares (LVSI), habitualmente sólo es posible de determinar en forma confiable en biopsia definitiva, no así en biopsia por congelación (14). Se ha demostrado que en pacientes con cáncer de endometrio clínicamente limitado al útero, la etapificación quirúrgica completa permite dirigir el uso de tratamiento adyuvante y mejora la sobrevida (15-18).

Nuestros resultados son concordantes con lo demostrado por Frumowitz y cols (1), Ben Schachar y cols (4), Case y cols (5), Fotiou y cols (19), Wang y cols (20), Kumar y cols (21), y en nuestro medio por Puga y cols (2), que demuestran que los parámetros histopatológicos pre e intraoperatorios analizados en nuestro estudio en un porcentaje significativo son distintos en la biopsia definitiva, por lo que recomiendan taxativamente no utilizarlos para decidir si efectuar o no la etapificación quirúrgica completa en pacientes con cáncer de endometrio clínicamente limitado al útero.

En base a nuestros resultados, a los de varios autores $(1,2,4,5,19-21)$, y a las recomendaciones de autores y autoridades internacionales y de nuestro medio $(3,10,16,17,22-24)$, estimamos que no debiera usarse la información de la histología preo- peratoria o de biopsia uterina intraoperatoria para decidir si efectuar o no la etapificación quirúrgica completa en pacientes con cáncer de endometrio.

\section{CONCLUSIÓN}

Producto del análisis de nuestros resultados y de los los numerosos autores citados que concuerdan con nuestros hallazgos (1-5,16,17,19-21), en nuestro servicio el protocolo de manejo de pacientes con cáncer de endometrio clínicamente limitado al útero, contempla actualmente realizar etapificación quirúrgica completa, a toda paciente que no presente comorbilidad que contraindique la cirugía.

\section{REFERENCIAS}

1. Frumovitz $M$, Singh DK, Meyer L, et al. Predictors of final histology in patients with endometrial cancer. Gynecol Oncol 2004;95:463-8.

2. Puga M, Prado S, Garrido J, et al. Correlación entre biopsia contemporánea y diferida en cáncer de endometrio en el INC. Estudio preliminar. XXX Congreso Chileno de Obstetricia y Ginecología. 2005. C-031.

3. Puente R, Israel E, Guzmán S, etal. La linfadenecto $\neg m i ́ a$ aórtica y pelviana en el carcinoma de endometrio. Rev Chil Obstet Ginecol 1996;61:332-40.

4. Ben-Shachar I, Pavelka J, Cohn DE, et al. Surgical staging for patients presenting with grade 1 endometrial carcinoma. Obstet Gynecol 2005;105:487-93.

5. Case AS, Roconni RP, Straughn JM Jr, et al. A prospective blinded evaluation of the accuracy of frozen section for the surgical management of endometrial cancer. Obstet Gynecol 2006;108:1375-9.

6. Rodríguez T, Yazigi R. Adenocarcinoma de endometrio. Etapa precoz y enfoque quirúrico. Rev Med Clin Condes 2006;17:76-9.

7. Nuñez F, Puga O, Brañes J, et al. Abordaje laparoscópico en cáncer de endometrio. Rev Chil Obstet Ginecol 2009;74:172-8.

8. Altman DG, Bland JM. Statistics Notes: Diagnostic tests 1: sensitivity and specificity. BMJ 1994;308:1552.

9. Altman DG, Bland JM. Statistics Notes: Diagnostic tests 2: predictive values. BMJ 1994;309:102.

10. Creasman WT, Morrow CP, Bundy BN. Surgical pathologic spread patterns of endometrial cancer. A Gynecologic Oncolgy Group study. Cancer 1987;60(8 suppl):2035-41.

11. Morow CP, Bundy BN, Kurman RJ, et al. Relationship between surgical-pathologic risk factors and outcome in clinical stage I and II carcinoma of the endometrium: a Gynecologic Oncology Group study. Gynecol Oncol 1991;40:55-65.

12. Larson DM, Connor GP, Broste SK, et al. Prognostic significance of gross myometrial invasión with endometrial cancer. Obstet Gynecol 1996;88:394-8.

13. Boronow RC, Morrow CP, Creasman WT, et al. Surgical staging in endometrial cancer: clinical-pathologic findings of a prospective study. Obstet Gynecol 
1984;63:825-32.

14. Inoue $\mathrm{Y}$, Obata K, Abe K, et al. Prognostic significance of vascular invasion by endometrial carcinoma. Cancer 1996;78:1447-51.

15. Chan JK, Kapp DS. Role of complete lymphadenectomy in endometrioid uterine cancer. Lancet Oncol 2007;8:831-41.

16. Chan JK, Cheung MK, Huh WK, et al: Therapeutic role of lymph node resection in endometrioid corpus cancer: A study of 12,333 patients. Cancer 2006;107:1823-30.

17. Chan JK, Sherman AE, Kapp DS, et al. Influence of gynecologic oncologists on the survival of patients with endometrial cancer. J Clin Oncol 2011;29:832-8.

18. Cragun JM, Havrilesky LJ, Calingaert B, et al. Retrospective analysis of selective lymphadenectomy in apparent early-stage endometrial cancer. J Clinical Oncology 2005;23:3668-75.
19. Fotiou S, Trimble EL, Papakonstantinou K, et al. Complete pelvic lymphadenectomy in patients with clinical early, grade I and II endometrioid corpus cancer. Anticancer Res 2009;29:2781-6.

20. Wang XY, Pan ZM, Chen XD, et al. Accuracy of tumor grade by preoperative curettage and associated clinicopathologic factors in clinical stage I endometrioid adenocarcinoma. Chinese Medical Journal 2009;122(16):1843-6.

21. Kumar S, Bandyopadhyay S, Semaan A, et al. The role of frozen section in surgical staging of low risk endometrial cancer. PLoS ONE 2011;6(9):e21912.

22. Chalas $E$. Endometrial cancer: what is a clinician to do? Obstet Gynecol 2007;110:1222-3.

23. ACOG Committee on Practice Bulletins. Management of endometrial cancer 2005;65:1-9.

24. Kirby TO, Leath CA 3rd, Kilgore LC. Surgical staging in endometrial cancer. Oncology (Williston Park) 2006;20:45-50. 